\title{
Preferential Impairment of Avoidance Performances in Amygdala-Lesioned Mice
}

\author{
Ken Takashina, Hiroshi Saito and Nobuyoshi Nishiyama* \\ Department of Chemical Pharmacology, Faculty of Pharmaceutical Sciences, The University of Tokyo, Bunkyo-ku, Tokyo 113, Japan
}

Received August 22, $1994 \quad$ Accepted November 10, 1994

\begin{abstract}
To clarify the role of the amygdala in the fulfillment of memory and/or learning, amygdalalesioned mice were tested in passive and active avoidance performances and also in spatial learning tasks. Although the lesioned animals showed deteriorated performances in both passive and active avoidance tests, they executed the spatial learning tasks as well as the control mice. The learning deficit was prominent in the process of memory acquisition of passive and active avoidance tasks, suggesting that the amygdala might be involved in the acquisition processes of these avoidance tests. The locomotor activities of the lesioned animals were slightly increased, but there was no significant difference compared with the control mice. These findings indicate that the amygdala plays a crucial role preferentially in the avoidance learning rather than the spatial learning.
\end{abstract}

Keywords: Passive avoidance, Active avoidance, Spatial learning, Memory acquisition, Emotion

Numerous researches have suggested that central cholinergic systems play an important role in the process of learning and memory (see Ref. 1 for a review). The amygdala is one of the major target areas of the afferent projections from basal forebrain cholinergic neurons, and this system modulates certain kinds of learning performance via activation of the muscarinic and nicotinic receptors $(2-6)$. Our previous studies have also revealed that in passive and active avoidance tasks, cholinergic neurotransmission is activated in the amygdala of good learners as compared with that of poor learners in mice $(7-10)$. In addition, Nomura et al. (11) recently demonstrated that intra-amygdala injection of scopolamine deteriorated the passive avoidance performances in mice. These lines of evidence suggest that the amygdala, and probably the cholinergic transmission in the amygdala, is one of the indispensable components for the completion of learning and memory. In this paper, we analyzed the learning behaviors of amygdala-lesioned mice in duplicate sets of passive avoidance (step-through and stepdown tests), active avoidance (shuttle box and lever press tests), and spatial learning tasks (water maze and radialarm maze tests) to obtain a systematic and comprehensive understanding of the involvement of the amygdala in the learning behaviors of mice.

\footnotetext{
* To whom correspondence should be addressed.
}

\section{MATERIALS AND METHODS}

\section{Animals}

Male Std-ddY mice at the age of 8 weeks (SLC, Hamamatu) were used after 1 week acclimation to the breeding environment. They were housed in groups of 5 animals in metal cages $(30 \times 20 \times 10 \mathrm{~cm})$ with wooden chip flooring, and they had free access to food and water, except for the period of food restriction in the radial-arm maze test. The temperature and humidity of the room were controlled at $22 \pm 1^{\circ} \mathrm{C}$ and $55 \pm 5 \%$, respectively.

\section{Amygdala lesion}

Under anesthesia with a ketamine and xylazine mixture ( 80 and $7 \mathrm{mg} / \mathrm{kg}$, respectively, i.m.), the mice were placed on a stereotaxic apparatus (Narishige, Tokyo). They received bilateral amygdala lesions by a delivery of radiofrequency current using a lesion generator (Radionics, Inc., Burlington, MA, USA). The coordinates of the electrode placement used were: $0.7 \mathrm{~mm}$ posterior to the bregma, $\pm 2.7 \mathrm{~mm}$ lateral to the sagital suture and $0.7 \mathrm{~mm}$ below the ear-bar's 0 point, with the incisor bar placed at $2.2 \mathrm{~mm}$ ventral to the horizontal plane (12). The stainless steel electrode $(0.25-\mathrm{mm}$ diameter) was inserted into the brain, and the tip of it was heated at $70^{\circ} \mathrm{C}$ for $60 \mathrm{sec}$. The inserted electrode was not heated in the sham-operated group of mice. Age matched intact mice were used as the 
control group. After the mice had recovered from the operation, independent sets of mice were tested in the passive avoidance tasks (step-through and step-down tests), active avoidance tasks (shuttle box and lever press tests), spatial learning tasks (water maze and radial-arm maze tests), and motor activity measurement with a tilting type ambulometer. These behavioral tests were started two weeks after the surgery except for the radial-arm maze test that was started three weeks after the operation. Prior to the radial-maze test, mice were subjected to food intake restriction of $2 \mathrm{hr}$ a day at the same time of the day to maintain their body weight at approximately $85 \%$ of the free-feeding level. They were tested in the radial-maze test following one week on the food restriction schedule. Our preliminary results indicated that the threshold in pain sensitivity to an electric foot shock was not altered by amygdala-lesion.

\section{Step-through test}

A two-compartment step-through passive avoidance apparatus (PAM1; O'hara \& Co., Ltd., Tokyo) was used. Details of the device and procedures are described elsewhere (13). Briefly, in the learning trial, each mouse was placed in the bright compartment of the apparatus. When the mouse entered into a dark compartment, an electric shock (max $0.2 \mathrm{~mA}, 36 \mathrm{~V} \mathrm{AC}$ ) was delivered automatically through the grid floor and the mouse escaped back to the bright compartment. The time elapsed to enter the dark compartment was recorded as "latency". The number of animals that entered the dark compartment in the first testing trial was also recorded. From the following day, the mice were submitted to testing trials for a consecutive 6 days at the same time of day using the same procedure as in the learning trial. The ceiling time for the latency to enter the dark compartment was set at $300 \mathrm{sec}$. A mouse that did not enter the dark chamber for the first time in the testing trials was regarded as an animal that had attained the criterion of memory acquisition. An averaged number of testing trials required to reach this criterion of memory acquisition was calculated and expressed as "days for acquisition". The total number of days on which mice entered the dark compartment and received an electric shock during the testing trials was defined as the "total number of errors". Ten mice were used in one experimental group.

\section{Step-down test}

The apparatus for the step-down test consisted of a chamber equipped with a grid floor and an escaping platform located in one corner of the chamber. Details of the device and procedures are elsewhere (13). In the learning trial, each mouse was placed on the platform and allowed to explore the apparatus for $10 \mathrm{~min}$. An electric current was continuously sent to the grid floor of the apparatus. When the mouse got off from the platform, a punishing foot shock (60V AC) was applied through the floor grid. During the latter half $(5 \mathrm{~min})$ of the learning trial, the number of the shocks received by a descent from the platform was recorded as the "number of stepping down" or "error". Testing trials were given for another 6 days at the same time of day using the same procedure as in the learning trial except that the observation time was $3 \mathrm{~min}$ in the testing trials. The number of animals that stepped down from the platform in the learning and in the first testing trials were recorded as the "number of animals with an error". A mouse that did not step down onto the grid floor for the first time in the testing trials was regarded to have attained the criterion for memory acquisition. An averaged number of testing trials required to reach this criterion of memory acquisition was calculated and expressed as "days for acquisition". The accumulated number of errors during the whole period of testing trials was defined as the "total number of errors". Ten mice were used in one experimental group.

\section{Shuttle box test}

Acquisition of the two-way avoidance response was assessed with an automated shuttle box (GT-8332S, O'hara \& Co., Ltd.) as previously described (14). The temporal parameters were as follows: intertrial interval of $40 \mathrm{sec}$; warning with a beep and flash of light $(30 \mathrm{msec}, 1 \mathrm{~Hz})$ of $20 \mathrm{sec}$; electric shock ( $36 \mathrm{~V} \mathrm{AC}$ ) of $10-\mathrm{sec}$ duration in the latter half of the warning period. An avoidance from the electric shock was attained by moving from one side of the apparatus to the other in the former (conditioned avoidance) or the latter (unconditioned avoidance) half of the warning period. The indices of avoidance behavior were the conditioned avoidance rate (CAR: the number of conditioned avoidance responses/the number of the trials $\times 100$ ) and unconditioned avoidance rate (UAR: the number of unconditioned avoidance responses/the number of the trials $\times 100$ ). When the mice failed to avoid the electric shocks, that trial was regarded as an error. The number of movements not related to avoidance responses was counted as the spontaneous response. Each session consisted of 60 trials per day, and the sessions were performed for 5 consecutive days at the same time of day. Ten mice were used in one experimental group.

\section{Lever press test}

Acquisition of the lever press type avoidance response was determined in an automated lever press test apparatus (GT-8332, O'hara \& Co., Ltd.) as previously described (14). In this test, mice learned to avoid the electric shocks by pressing the lever hung on the shorter side of the wall of the apparatus in the warning period. The 
temporal parameters, intensity of electric shock and indices of avoidance response were similar to those of the shuttle box test. The number of lever pressings, which were not related to avoidance responses, were counted as the spontaneous response. Each session consisted of 60 trials per day, and sessions were performed for 6 consecutive days at the same time of day. Ten mice were used in one experimental group.

\section{Water maze test}

The pool for the water maze test was a circular blue plastic tank, $80 \mathrm{~cm}$ in diameter and $32 \mathrm{~cm}$ in depth, and filled to a height of $26 \mathrm{~cm}$ with tap water at $21 \pm 1^{\circ} \mathrm{C}$. A transparent platform $(10 \mathrm{~cm}$ in diameter) was placed at a constant location, $1 \mathrm{~cm}$ below the surface of the water, throughout a testing period of 5 consecutive days except day 6 when a visible black platform, fixed $0.5 \mathrm{~cm}$ above the surface of the water, was used to examine the eye sight and swimming ability of the mice. Several cues were arranged around the pool, which were visible to the swimming mice. The locations of these cues were kept unchanged throughout the period of the trials. Prior to the actual test, each mouse was trained to swim for $60 \mathrm{sec}$ to get accustomed to the experimental procedures. The scheme of the actual task was as follows: a mouse was put into the pool from one of the four starting points which divided the pool into four quadrants. The time for a mouse to find and get onto the platform was recorded as the "latency". After reaching the platform, a mouse was allowed to stay on it for $60 \mathrm{sec}$ to get a sense of spatial orientation. When the mouse failed to find the hidden platform within $90 \mathrm{sec}$, it was placed on the platform and forced to stay there for $60 \mathrm{sec}$ as well. The intertrial interval was set at $5 \mathrm{~min}$. The trials were performed for 6 consecutive days. Fifteen mice were used in one experimental group.

\section{Radial-arm maze test}

The platform for the maze was elevated $60 \mathrm{~cm}$ above the floor. Eight arms $(5.5 \mathrm{~cm} \times 30 \mathrm{~cm})$ extended from an octagonally shaped central hub. A small cup $(2.7 \mathrm{~cm}$ in diameter), served as a receptacles for rewarding pellets (20 $\mathrm{mg} /$ pellet, O'hara \& Co., Ltd.), were made at each end of the arm. The automated tracing system for animal behavior (BTA-2A; Muromachi, Tokyo) was set up over the maze. Mice were given free exploration of the maze for 5 min prior to the actual testing. At the beginning of each testing trial, food pellets were placed in all cups. A mouse was gently placed on the central hub and allowed to have free access to all arms. The trial was ended when the mice had made 8 choices in total or spent $10 \mathrm{~min}$ in the maze. An entry into an arm for the first time in a daily session was recorded as a correct choice, and a re-entry was consi- dered to be an error. The number of correct choices before committing the first error was counted as the "initial correct choice" and served as an index of the maze learning. The total time spent in the trial and average speed of movement were also recorded as parameters of the kinetic activities of the mice. The tests were performed for 9 consecutive days at the same time of day. The observed parameters were combined every three days and expressed as a "block". Blocks 1, 2 and 3 represent the result of days $1-3,4-6,7-9$, respectively. Eight mice were used in one experimental group.

\section{Locomotor activity test}

A mouse was placed in a round testing cage $(19 \mathrm{~cm}$ in diameter and $18 \mathrm{~cm}$ high), which was supported at the center of its bottom and three points around it ( $\mathrm{O}^{\prime}$ hara \& Co., Ltd.). When the mouse moved, the cage tilted toward one or two side supports, operating switches to count the movements of the mouse. Each mouse was measured for its locomotor activity for $30 \mathrm{~min}$. The test was performed for 6 consecutive days at the same time of the day. Ten mice were used in one experimental group.

\section{Histology}

Following the completion of the behavioral test, the brains of amygdala-lesioned animals were removed and fixed with $4 \%$ paraformaldehyde solution. Coronal sections of $50 \mu \mathrm{m}$ were made with a frozen-stage microtome and stained with cresyl violet. The location of the lesioned site in the amygdala was verified under a light microscope.

\section{Statistics}

Comparison of the number of animals that entered into the dark compartment or made errors in the step through or step down test, respectively, was done with the SteelDwass test (multiple comparison of frequency). The data on body weight was analyzed with ANOVA and post hoc Duncan's multiple range test. All other data were analyzed with the Kruskal-Wallis test (non-parametric multiple comparison) followed by Mann-Whitney's $U$-test.

\section{RESULTS}

\section{Change in the body weight}

The body weight of the lesioned mice decreased steeply during the two days after the surgery and then recovered slowly. The gain of body weight in the lesioned group was comparable to those of the control or sham-operated group at two weeks after the operation (Table 1). Although the body weight in the lesioned mice was less than those of the control or sham-operated group at any time point, it recovered to the level at the time of the oper- 
Table 1. Changes in the body weight of mice after bilateral amygdala lesion

\begin{tabular}{|c|c|c|c|c|c|}
\hline & \multirow[b]{2}{*}{ Operation } & \multicolumn{4}{|c|}{ Days after operation } \\
\hline & & 2 & 7 & 14 & 20 \\
\hline CONT & $34.4 \pm 0.3$ & $35.2 \pm 0.4$ & $37.3 \pm 0.5$ & $37.4 \pm 0.6$ & $37.9 \pm 0.5$ \\
\hline SHAM & $34.6 \pm 0.2$ & $33.9 \pm 0.2$ & $36.6 \pm 0.4$ & $36.4 \pm 0.6$ & $37.6 \pm 0.8$ \\
\hline LESION & $34.4 \pm 0.3$ & $30.6 \pm 0.7^{* *}$ & $34.1 \pm 0.4^{* *}$ & $34.3 \pm 0.5^{*}$ & $35.0 \pm 0.4^{* *}$ \\
\hline
\end{tabular}

Each value represents the mean \pm S.E.M. of ten mice. CONT: control group, SHAM: sham-operated group and LESION: amygdala-lesioned group. Statistical significance by ANOVA and post hoc Duncan's multiple range test is shown as ${ }^{*} \mathrm{P}<0.05$, ${ }^{* *} \mathrm{P}<0.01$ vs sham-operated group.

ation by two weeks after the surgery.

\section{Step-through test}

The mean latency to enter the shock compartment in the learning trial was not different among the groups, suggesting that locomotor activity was not altered by amygdala lesion. In the first testing trial, the latency was significantly shorter in the lesioned animals as compared with the control or sham-operated mice. In the first testing trial, almost no mice stepped through into the shock compartment but stayed in the bright chamber for the maximum time of observation $(300 \mathrm{sec})$ in the control and sham-operated mice. However, in the amygdala-lesioned group, $70 \%$ of the mice entered into the shock chamber within $300 \mathrm{sec}$ (Table 2). Moreover, lesioned animals took significantly more days to reach the criterion of memory acquisition than the control or sham operated mice. The total number of errors during the whole testing trial was also increased in the lesioned animals as compared with those in the control or sham-operated mice.

\section{Step-down test}

The number of errors in the learning as well as in the first testing trials tended to increase in the lesioned mice, but it did not reach statistical significance. There was also no significant differences in the number of animals with errors among the tested groups. Although almost all mice in the control and sham-operated group reached the criterion of memory acquisition by the time of the second testing trial, the amygdala-lesioned mice required more than four trials (Table 2). The total number of errors during the period of the testing trials were significantly increased in the lesioned animals as compared with these of the control and sham-operated animals.

\section{Shuttle box test}

Control and sham-operated mice gained a conditioned avoidance rate quite rapidly in the shuttle box test (Fig. 1A). Eighty percent of the trials were successful as early as day 2. The amygdala-lesioned mice showed a significantly slower memory registration than the sham-oper-

Table 2. Effects of amygdala lesion on learning behaviors in the step-through and step-down tests Step-through test

\begin{tabular}{|c|c|c|c|c|c|c|}
\hline \multirow{2}{*}{ Group } & \multirow{2}{*}{$\begin{array}{l}\text { No. of } \\
\text { animals }\end{array}$} & \multicolumn{2}{|c|}{ Latency $(\mathrm{sec})$} & \multirow{2}{*}{$\begin{array}{l}\text { No. of entered } \\
\text { animals (Day 1) }\end{array}$} & \multirow{2}{*}{$\begin{array}{l}\text { Days for } \\
\text { acquisition }\end{array}$} & \multirow{2}{*}{$\begin{array}{l}\text { Total No. } \\
\text { of errors }\end{array}$} \\
\hline & & Learning trial & Testing trial (Day 1) & & & \\
\hline CONT & 10 & $11.0 \pm 1.7$ & $300.0 \pm 0.0$ & 0 & $1.0 \pm 0.0$ & $0.8 \pm 0.4$ \\
\hline SHAM & 10 & $12.2 \pm 2.1$ & $283.4 \pm 16.6$ & 1 & $1.1 \pm 0.1$ & $1.4 \pm 0.3$ \\
\hline LESION & 10 & $13.1 \pm 2.7$ & $131.8 \pm 42.9^{*, a)}$ & $7^{*, \mathrm{~b})}$ & $3.4 \pm 0.7^{*, \mathbf{a})}$ & $3.3 \pm 0.6^{* . a)}$ \\
\hline \multicolumn{7}{|c|}{ Step-down test } \\
\hline \multirow{2}{*}{ Group } & \multirow{2}{*}{$\begin{array}{l}\text { No. of } \\
\text { animals }\end{array}$} & \multicolumn{2}{|c|}{ No. of stepping down } & No. of entered & Days for & Total No. \\
\hline & & Learning trial & Testing trial (Day 1) & animals (Day 1) & acquisition & of errors \\
\hline CONT & 10 & $0.9 \pm 0.4$ & $0.5 \pm 0.2$ & 4 & $2.1 \pm 0.6$ & $4.2 \pm 1.9$ \\
\hline SHAM & 10 & $1.5 \pm 0.7$ & $1.4 \pm 0.6$ & 6 & $2.0 \pm 0.3$ & $3.5 \pm 0.9$ \\
\hline LESION & 10 & $3.4 \pm 0.9$ & $2.0 \pm 0.5$ & 8 & $\left.4.5 \pm 0.7^{*}, \mathrm{a}\right)$ & $\left.9.4 \pm 1.1^{* *}, a\right)$ \\
\hline
\end{tabular}

Each value represents the mean \pm S.E.M. CONT: control group, SHAM: sham-operated group and LESION:

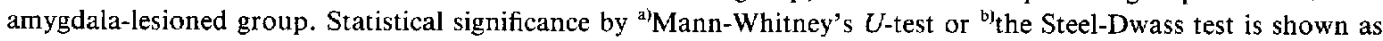
${ }^{*} \mathrm{P}<0.05,{ }^{* *} \mathrm{P}<0.01$ vs sham-operated group. 

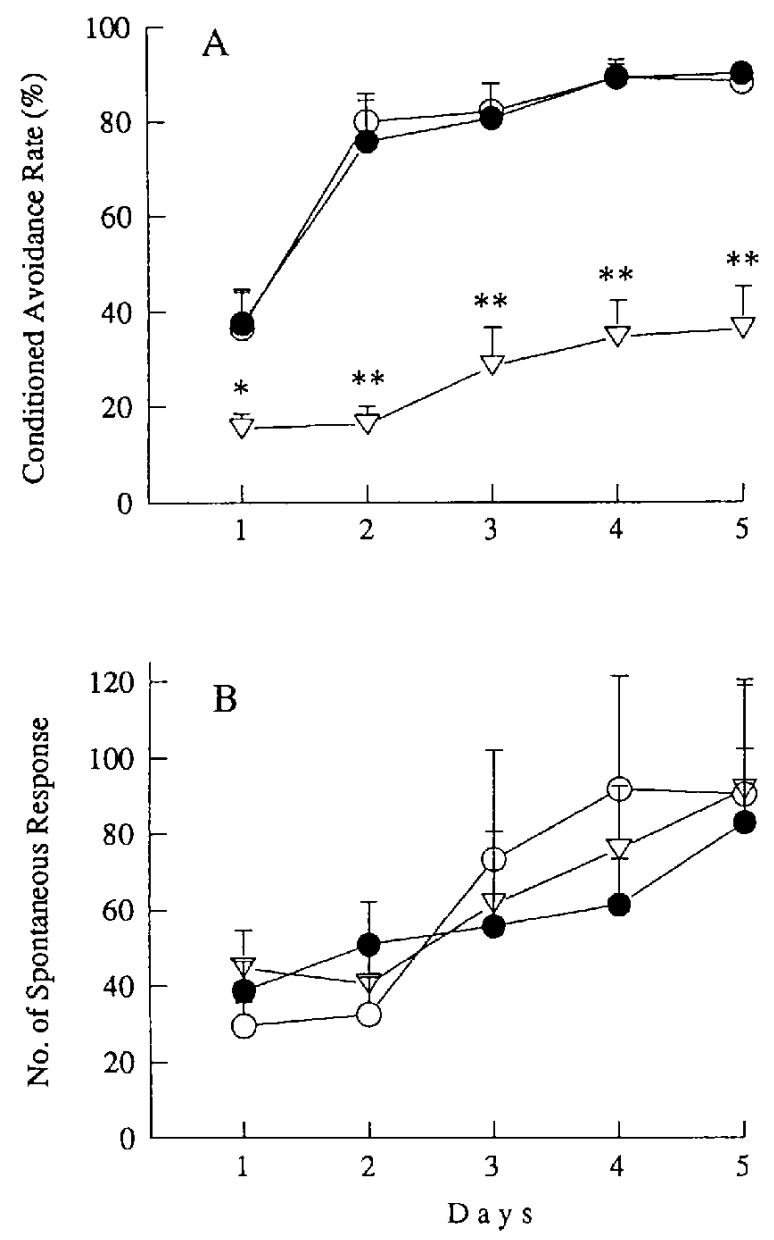

Fig. 1. Effects of amygdala-lesion on behavioral performances in shuttle box test. The conditioned avoidance rate (A) and the number of spontaneous response (B) were recorded daily for 5 days. Results of the control (open circle), sham-operated (closed circle) and amygdala-lesioned group (open triangle) are shown as the mean \pm S.E.M. of ten mice. ${ }^{*} \mathbf{P}<0.05,{ }^{* *} \mathrm{P}<0.01$ vs sham-operated mice by Mann-Whitney's $U$-test.

ated ones in terms of this parameter. Only $40 \%$ of the trials were successful in the final session of day 5. The number of spontaneous responses, which is not related to an avoidance or an escaping reaction, increased similarly in the control, sham-operated and amygdala-lesioned mice (Fig. 1B). The unconditioned avoidance rate delineated an inverse pattern of conditioned avoidance rate in all the tested groups (data not shown). A correlation analysis between the conditioned avoidance rate and the number of spontaneous responses in the shuttle box test revealed a significant correlation of the two parameters in the lesioned group in all daily sessions (Table 3 ).

\section{Lever press test}

Control and the sham-operated animals slowly but steadily increased their conditioned avoidance rate. In the
Table 3. Correlation analysis between conditioned avoidance rate and spontaneous response in the active avoidance tasks

Shuttle box test

\begin{tabular}{lccccc}
\hline & day 1 & day 2 & day 3 & day 4 & day 5 \\
\hline CONT & 0.66 & 0.39 & 0.50 & 0.32 & 0.58 \\
SHAM & 0.42 & 0.59 & 0.47 & 0.49 & 0.60 \\
LESION & $0.96^{\# \#}$ & $0.95^{\# \#}$ & $0.91^{\# \#}$ & $0.95^{\# \#}$ & $0.97^{\# \#}$ \\
\hline
\end{tabular}

Lever press test

\begin{tabular}{lcccccc}
\hline & day 1 & day 2 & day 3 & day 4 & day 5 & day 6 \\
\hline CONT & $0.67^{\sharp}$ & 0.27 & 0.36 & -0.08 & -0.40 & -0.46 \\
SHAM & 0.45 & 0.46 & 0.46 & 0.32 & 0.39 & 0.37 \\
LESION & $0.67^{\sharp}$ & 0.36 & 0.40 & 0.36 & 0.31 & 0.06 \\
\hline
\end{tabular}

Each value represents the correlation coefficient between the conditioned avoidance rate and spontaneous response $(n=10)$. CONT: control group, SHAM: sham-operated group and LESION: amygdala-lesioned group. A statistically significant correlation is indicated by ${ }^{\#} \mathrm{P}<0.05,{ }^{* \#} \mathrm{P}<0.01$.

final testing session at day 6 , control and sham-operated mice succeeded in $71.5 \%$ and $65.2 \%$ of the trials, respectively. In contrast, lesion of the amygdala significantly inhibited the gain of conditioned avoidance rates in all daily sessions. Although almost no mice in the control (none out of ten) and sham-operated group (one out of ten) committed an error in the final session, six out of ten amygdala-lesioned mice made an error. No significant differences in the number of spontaneous responses were observed among the three tested groups (Fig. 2B). The conditioned avoidance rate and spontaneous response were statistically correlated only in the first session of the amygdalalesioned mice as well as the control mice, but no such correlation was present in the following sessions (Table 3 ).

\section{Water maze test}

Escaping latency to find a hidden platform decreased steeply in the first 2 days and thereafter gradually in the control and sham-operated groups. Bilateral lesion of the amygdala did not affect the decrease in the escaping latency in all daily sessions (Fig. 3). This was also the case when the platform was replaced with a black visible one above the surface of the water on day 6 .

\section{Radial-arm maze test}

Initial correct choice, the number of correct choices prior to the first commitment of an error, increased timedependently in both the control and sham-operated groups. Amygdala-lesion did not affect this parameter of initial correct choice in any block of the radial-arm maze test (Fig. 4). Moreover, the initial correct choice of the 

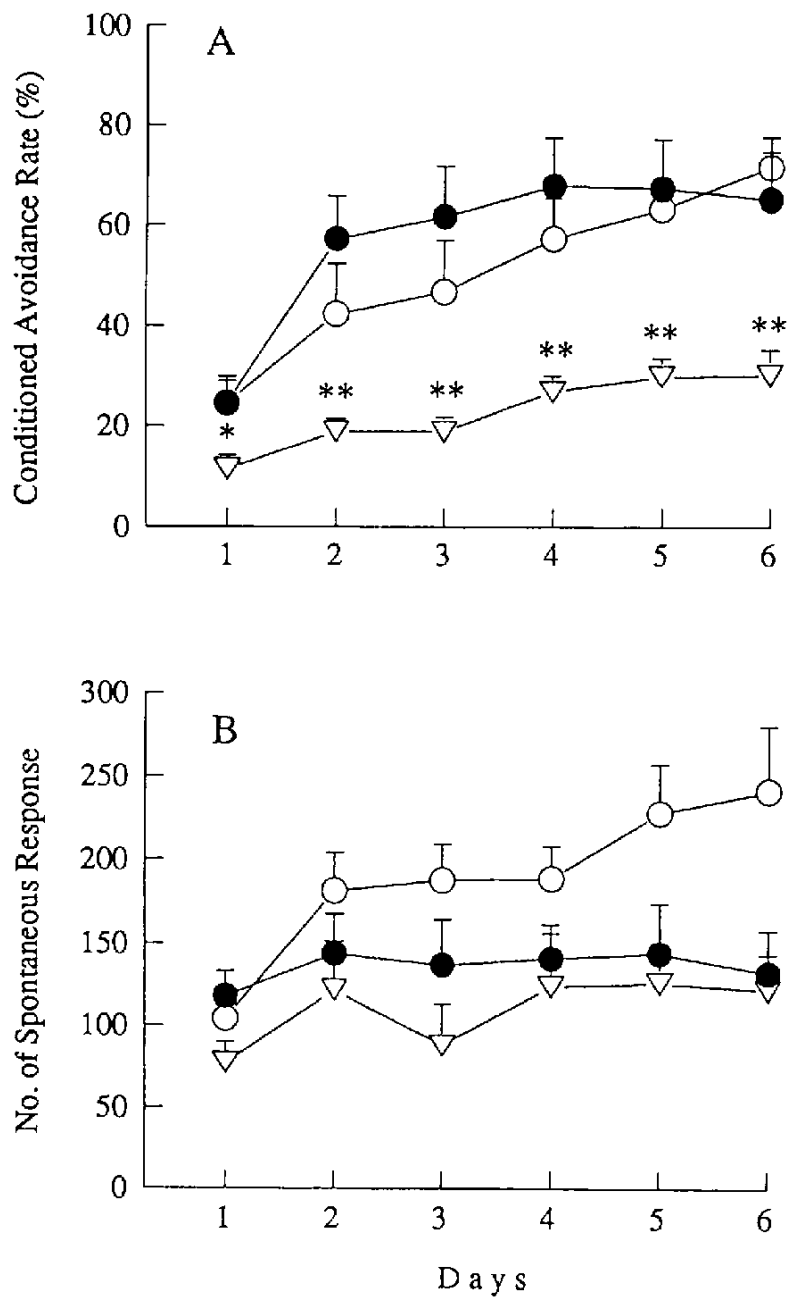

Fig. 2. Effects of amygdala-lesion on behavioral performances in the lever press test. The conditioned avoidance rate (A) and the number of spontaneous responses (B) were recorded daily for 6 days. Results of the control (open circle), sham-operated (closed circle) and amygdala-lesioned group (open triangle) are shown as the mean \pm S.E.M. of ten mice. ${ }^{*} \mathrm{P}<0.05,{ }^{* *} \mathrm{P}<0.01$ vs sham-operated mice by Mann-Whitney's $U$-test.

amygdala-lesioned mice was above the chance level of 3.22 , which is the calculated value when the mouse selected the arms at random, in all blocks. In contrast, the total time of movement was significantly increased by amygdala-lesion in every block (Table 4). The average speed of movement was decreased in the second block of the amygdala-lesioned mice as compared to that in the sham-operated ones, but the difference was not obvious in the other blocks (Table 4).

\section{Locomotor activity test}

Because the total time of movement was increased and the average speed was temporarily decreased by lesion of the amygdala in the radial-arm maze test, we measured

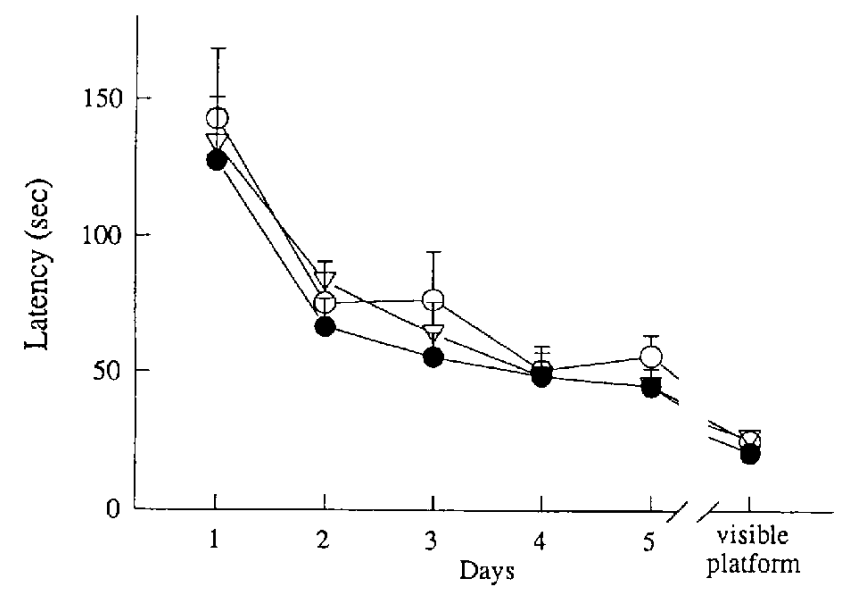

Fig. 3. Effects of amygdala-lesion on escaping latency in the Morris water maze test. Each mouse was subjected to four trials per day that started from four randomly assigned positions. The latency indicates the sum of the time required for a mouse to reach the platform in four trials. The platform used was transparent and hidden under the surface of the water in the trials through days 1 to day 5 , while it was changed into a black one above the surface of the water in the trial of day 6 as a visible platform test. Results of the control (open circle), sham-operated (closed circle) and amygdala-lesioned group (open triangle) are shown as the mean \pm S.E.M. of fifteen mice.

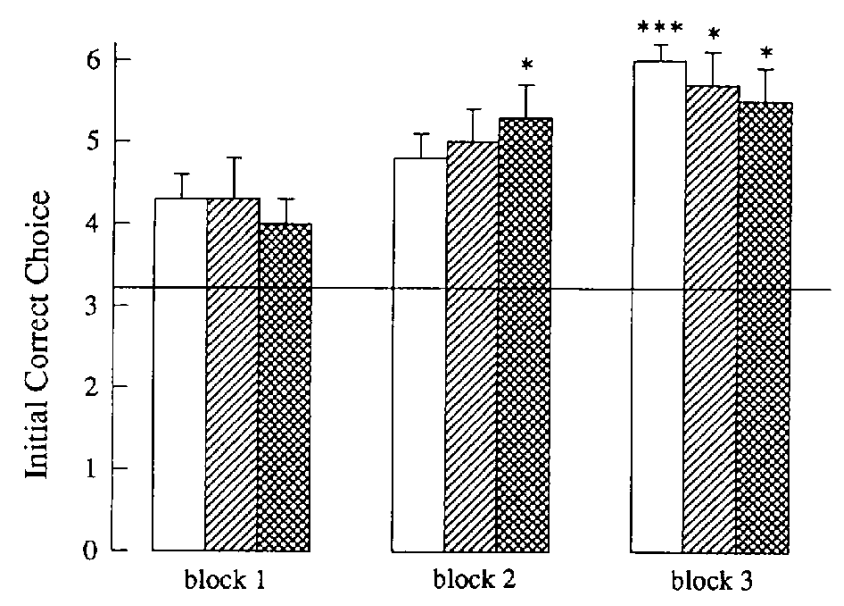

Fig. 4. Effects of amygdala-lesion on the initial correct choice in the radial-arm maze test. A mouse was allowed to have free access to all arms that contained food pellets. An entry into an arm for the first time in a daily session was recorded as a correct choice, and a reentry was considered to be an error. The number of correct choices before committing the first error was illustrated as the initial correct choice. The tests were performed for 9 consecutive days. The results were combined every three days and expressed as a block. The data of the control (blank column), sham-operated (hatched column) and amygdala-lesioned group (crosshatched column) are shown as the mean \pm S.E.M. of eight mice. A horizontal line at the value of 3.22 indicates the calculated chance level of the initial correct choice. ${ }^{*} \mathrm{P}<0.05,{ }^{* * *} \mathrm{P}<0.001$ vs the data of block 1 in the corresponding group (Mann-Whitney's $U$-test). 
Table 4. Effects of amygdala-lesion on kinetic activities of mice in radial-arm maze test

Total time (sec)

\begin{tabular}{|c|c|c|c|}
\hline & block 1 & block 2 & block 3 \\
\hline CONT & $218 \pm 21$ & $179 \pm 26$ & $178 \pm 22$ \\
\hline SHAM & $221 \pm 32$ & $145 \pm 20$ & $163 \pm 16$ \\
\hline LESION & $294 \pm 23^{* *}$ & $285 \pm 26^{* *}$ & $297 \pm 25^{* *}$ \\
\hline \multicolumn{4}{|c|}{ Average of speed $(\mathrm{cm} / \mathrm{sec})$} \\
\hline & block 1 & block 2 & block 3 \\
\hline CONT & $23.9 \pm 0.4$ & $24.1 \pm 0.5$ & $25.0 \pm 0.3$ \\
\hline SHAM & $24.0 \pm 0.4$ & $24.9 \pm 0.5$ & $24.1 \pm 0.5$ \\
\hline LESION & $23.3 \pm 0.4$ & $23.3 \pm 0.2^{* *}$ & $23.6 \pm 0.6$ \\
\hline
\end{tabular}

Each value represents the mean \pm S.E.M. of eight animals. CONT: control group, SHAM: sham-operated group and LESION: amygdala-lesioned group. Statistical significance by MannWhitney's $U$-test is indicated by ${ }^{* *} \mathrm{P}<0.01 \mathrm{vs}$ sham-operated group.

the spontaneous motor activity of the amygdala-lesioned mice with a tilting type ambulometer for 7 days (Fig. 5). Amygdala-lesion tended to increase the spontaneous motor activity as compared with the activity in the control and sham-operated mice, but there was no statistical significance throughout the period of testing.

\section{Histology}

In the lesioned brain, extensive gliosis and numerous cells with small nuclei were observed under a light microscope in and around the amygdaloid subnuclei including the medial, lateral, basolateral and central nucleus at two

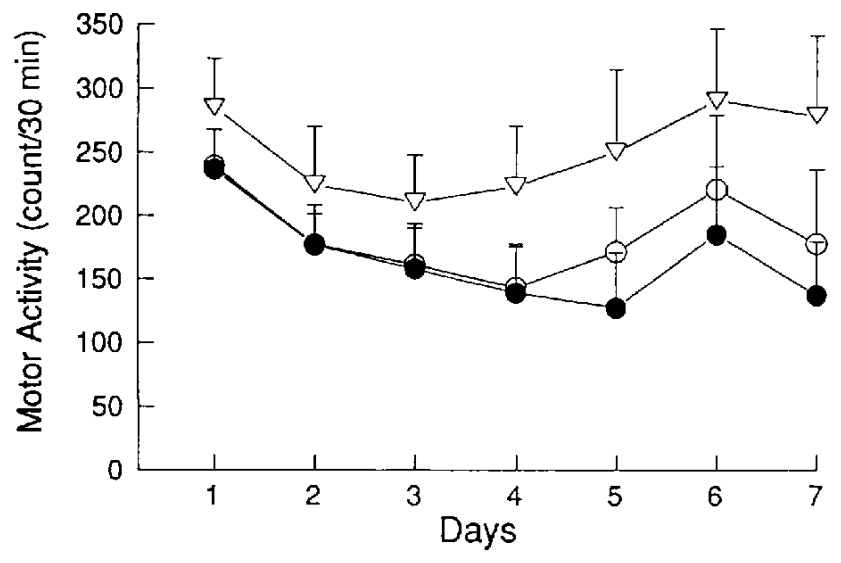

Fig. 5. Effects of amygdala-lesion on motor activity. Motor activity was measured with a tilting type ambulometer for $30 \mathrm{~min}$ every day for 7 consecutive days. Results of the control (open circle), sham-operated (closed circle) and amygdala-lesioned group (open triangle) are shown as the mean \pm S.E.M. of ten mice. weeks after the operation, which was still observed 40 days after the lesion (data not shown).

\section{DISCUSSION}

The amygdala consists of several subnuclei, and their functions are supposed to be different from each other (15). Because it was not practicable to produce a lesion in a specific subnuclei in mice, we destroyed the whole amygdala completely by thermolytic lesion. The radio-frequency current method employed in this study demolishes not only the cell body but also the passage fibers of neurons in the lesioned area. Although neurotoxic lesioning with chemicals such as ibotenic or kainic acids has an advantage in terms of selective damage to the neuronal cell bodies (16), we thought it would be difficult to produce uniform damage to a desired brain area because of its diffusiveness, especially when small animals such as mice are utilized. The body weight of the lesioned mice decreased for a few days after the operation, but it recovered to the pre-operation level in a couple of weeks, suggesting that the direct effect of the operational invasion had disappeared by this time. Therefore, we started the behavioral tests or food restriction two weeks after the surgery.

Control and sham-operated mice performed the stepthrough or step-down test successfully by the time of the first or second testing trial, respectively. Lesion of the amygdala induced a significant delay in accomplishing these tasks. Furthermore, amygdala-lesioned mice showed more errors not only in the initial testing trials but also in the learning trial in the step-down test. These results suggest that amygdala-lesion disrupted the acquisition processes in the passive avoidance tasks. Moreover, amygdala-lesion inhibited an increase of the conditioned avoidance rate in the shuttle box and lever press tests of active avoidance tasks. Because this parameter of conditioned avoidance rate is regarded to represent learning abilities, our results suggest that amygdala-lesion also impaired learning processes in active avoidance tests. The number of spontaneous responses was recorded in active avoidance tests. We had assumed that spontaneous response, which is not induced by conditioned or unconditioned foot shock, may reflect a certain aspect of locomotor activity and also the emotional status of the recipient mice as well. In order to get an insight into the specificity of the conditioned avoidance response as an index of learning ability, the values of the correlation coefficient were analyzed between the conditioned avoidance rate and the number of spontaneous responses. In the shuttle box test, no significant correlation was found between these two parameters in the control and sham-operated groups. These results suggest that the in- 
crease in conditioned avoidance rate observed in naive mice is not a secondary phenomenon evoked by a change of the spontaneous response, but reflect their learning abilities more directly. The conditioned avoidance rate of the amygdala-lesioned group, on the contrary, showed a significant positive correlation with the number of spontaneous responses. Since the number of spontaneous responses was indistinguishably similar between the amygdala-lesioned mice and control mice, a slight increase in the conditioned avoidance rate over the period of testing sessions in the lesioned animals might have been a secondary alteration induced by the increase of the spontaneous response. In the lever press test, there was no significant correlation between the conditioned avoidance rate and the number of spontaneous responses in all groups with a minor exception at the initial stage of the testing sessions. This indicates that the changes of the conditioned avoidance rate primarily represent alterations of learning capabilities in the lever press test.

Mean escaping latency to find a hidden platform in the Morris water maze test were not different among the control, sham-operated and amygdala-lesioned mice. In addition, bilateral lesion of the amygdala in mice did not affect the initial correct choice in the radial-arm maze test. These results indicate that amygdala-lesion does not interfere with spatial learning performances. Wieble et al. also reported that spatial alterations were not impaired by amygdala-lesion (17). In the radial maze test, the average speed of movement was statistically decreased in lesioned mice by comparison with the sham-operated group in the second block. However, the calculated difference was only temporal, and the actual values of average speed in lesioned mice were almost identical throughout the blocks, allowing us to speculate that there was virtually no change in the movement speed by amygdala-lesion. On the contrary, the total movement time in the radial-arm maze test was consistently higher in amygdala-lesioned mice than those in the control or sham-operated mice. Taking the result of movement speed into consideration, this observation indicates that amygdala-lesion halted or immobilized the mice during the maze trial more frequently or longer than the control mice. However, the spontaneous motor activity measured with a tilting type apparatus was not affected by amygdala-lesion. It is plausible that certain types of emotional alterations are induced by amygdala-lesion, but additional investigations will elucidate the underlying mechanism of this phenomenon.

An emotional motivation is considered to be indispensable to memory formation (10). In the present study, the task performances impaired by amygdala-lesion requires the animals to learn some new conditions or new rules with a motivation based on avoiding aversive stimuli. Indeed, the amygdala plays a critical role in associating aver- sive events with conditioned stimuli and regulating acquisition of conditioned fear $(18,19)$. Other investigators reported that amygdala-lesion after the formation of learning tasks utilizing aversive motivation did not induce significant disruption of its retention and retrieval (20). These findings suggest that the acquisition process might be primarily destroyed by amygdala-lesion in these learning tasks. On the other hand, the spatial learning tasks were not affected by the amygdala-lesion, suggesting that the mechanisms involved in the spatial learning are different from those of the avoidance learning and that the amygdala is not an consequential structure in executing these tasks. Ohno et al. reported that amygdala lesion in rats disrupted working but not reference memory in their original three panels runway test (21). Because it is difficult to discriminate working memory from reference memory in our task procedure, further analysis will be required in mice.

The present study provides conspicuous evidence that the amygdala plays an crucial role preferentially in avoidance learning when compared with spatial memory. These results agree well with those obtained in similar experiments in rats (6). On the contrary, the spatial learning performance is preferentially deteriorated when the septo-hippocampal cholinergic projection was damaged $(3,22,23)$. These results indicate that cholinergic activity in the amygdala, but not in the hippocampus, is more crucially involved in the passive avoidance performance than spatial cognition, and vice versa. Although the neurochemical mechanisms underlying the learning and memory is not yet completely resolved, we already found that the learning abilities of mice and cholinergic markers were significantly correlated in the amygdala (9-11). Moreover, Riekkinen et al. reported that the learning impairment induced by scopolamine (a muscarinic antagonist) or mecamylamine (a nicotinic antagonist) was alleviated by amygdala-lesion (6). In addition, intra-amygdala injection of norepinephrine produced memory enhancement in a lower dose and memory impairment at a high dose (24). These results indicate that cholinergic and/or noradrenergic projection to the amygdala modulates the passive avoidance performance in rodents. The amygdala is built in a complex neuronal network that seems to be profoundly involved in memory acquisition of avoidance learning, but the possibility cannot be ruled out that the amygdala plays only a supportive role in the memory processes that have or will be developed in other regions.

\section{REFERENCES}

1 Fibiger HC: Cholinergic mechanisms in learning, memory and dementia: a review of recent evidence. Trends Neurosci 14, $220-223(1991)$ 
2 McGaugh JL, Introini-Collison IB, Cahill LF, Castellano C, Dalmaz C, Parent MB and Williams CL: Neuromodulatory systems and memory storage: role of the amygdala. Behav Brain Res 58, $81-90$ (1993)

3 Izquierdo I, Medina JH, Bianchin M, Walz R, Zanatta MS, DaSilva RC, Bueno-e-Silva M, Ruschel AC and Paczko N: Memory processing by the limbic system: role of specific neurotransmitter systems. Behav Brain Res 58, 91 -98 (1993)

4 Dalmaz C, Introini-Collison IB and McGaugh JL: Noradrenergic and cholinergic interactions in the amygdala and the modulation of memory storage. Behav Brain Res 58, $167-174$ (1993)

5 Hughes $P$ and Dragunow $M$ : Muscarinic receptor mediated induction of Fos protein in rat brain. Neurosci Lett 150, 122126 (1993)

6 Riekkinen P Jr, Riekkinen $\mathbf{M}$ and Sirvio J: Cholinergic drugs regulate passive avoidance performances via the amygdala. J Pharmacol Exp Ther 267, 1484 - 1492 (1993)

7 Segawa, $\mathbf{M}$, Saito $\mathbf{H}$ and Nishiyama $\mathbf{N}$ : Alterations in choline acetyltransferase and tyrosine hydroxylase activities of various brain areas. Biogenic Amines 7, 171 - 180 (1990)

8 Segawa M, Saito $\mathrm{H}$ and Nishiyama N: Alterations in acetylcholine content and muscarinic cholinergic binding of various brain areas after the acquisition of active avoidance tasks in mice. Biogenic Amines 7, 181-190 (1990)

9 Segawa $M$, Saito $H$ and Nishiyama N: Alteration in choline acetyltransferase and tyrosine hydroxylase activities of various brain regions after passive avoidance performance in mice. Biogenic Amines 7, 191 - 197 (1990)

10 Saito $H$, Nishiyama $N$ and Segawa $M$ : Learning stress and the brain cholinergic system. In Stress: Neuroendocrine and Molecular Approaches, Edited by Kvetnansky R, McCarty R and Axelrod J, pp 99-108, Gordon and Breach Science Publishers SA, New York (1992)

11 Nomura $Y$, Nishiyama N, Saito $H$ and Matsuki N: Role of cholinergic neurotransmission in the amygdala on performances of passive avoidance learning in mice. Biol Pharm Bull 17, 490-494 (1994)

12 Sidman RL, Angevine JB Jr and Pierce ET: Atlas of the Mouse Brain and Spinal Cord. Harvard University Press, Cambridge (1971)
13 Zhang $\mathrm{Y}$, Saito $\mathrm{H}$ and Nishiyama N: Thymectomy-induced deterioration of learning and memory in mice. Brain Res 658, $127-134$ (1994)

14 Nishiyama N, Zhou Y, Takashina K and Saito H: Effects of DX-9386, a traditional Chinese prescription, on passive and active avoidance performances of mice. Biol Pharm Bull 17, 1472-1476 (1994)

15 Sarter M and Markowitsch $\mathbf{H J}$ : Involvement of the amygdala in learning and memory: A critical review, with emphasis on anatomical relations. Behav Neurosci 99, 342-380 (1985)

16 Ishihara $A$, Saito $H$, Ohta $H$ and Nishiyama $N$ : Basal forebrain lesioned mice exhibit deterioration in memory acquisition process in step through passive avoidance test. Jpn J Pharmacol 57, $329-336$ (1991)

17 Wible CG, Shiber IR and Olton DS: Hippocampus, fimbriafornix, amygdala and memory: Object discriminations in rats. Behav Neurosci 106, $751-761$ (1992)

18 Miserendino MJD, Sananes CB, Melia KR and Davis M: Blocking of acquisition but not expression of conditioned fear-potentiated startle by NMDA antagonists in the amygdala. Nature 345, 716-718 (1990)

19 Davis M: The role of the amygdala in fear and anxiety. Annu Rev Neurosci 15, 353-375 (1992)

20 Parent MB, Tomaz C and McGaugh JL: Increased training in an aversively motivated task attenuates the memory-impairing effects of posttraining $N$-methyl-D-aspartate-induced amygdala lesions. Behav Neurosci 106, 787-797 (1992)

21 Ohno $\mathrm{M}$, Yamamoto $\mathrm{T}$ and Watanabe $\mathrm{S}$ : Involvement of cholinergic mechanisms in impairment of working memory in rats following basolateral amygdaloid lesions. Neuropharmacology 31, 915-922 (1992)

22 O'keefe $\mathrm{J}$ and Dostrovsky J: The hippocampus as a spatial map. Brain Res 34, 171-175 (1971)

23 Olton DS, Becker JT and Handelman GE: Hippocampus space and memory. Behav Brain Sci 2, 313-316 (1979)

24 Liang KC, McGaugh JL and Yao HY: Involvement of amygdala pathways in the influence of the post-training intra-amygdala norepinephrine and peripheral epinephrine on memory storage. Brain Res 508, 225-233 (1990) 